\title{
A road traffic noise pattern simulation model that includes distributions of vehicle sound power levels
}

\author{
Bert De Coensel $^{\mathrm{a}, *}$, A. L. Brown ${ }^{\mathrm{b}}$, Deanna Tomerini ${ }^{\mathrm{b}}$ \\ ${ }^{a}$ Acoustics Group, Department of Information Technology, Ghent University, Belgium \\ ${ }^{b}$ Urban Research Program, Griffith School of Environment, Griffith University, Brisbane, Australia
}

\begin{abstract}
Annoyance, sleep disturbance and other health effects of road traffic noise exposure may be related to both level and number of noise events caused by traffic, not just to energy equivalent measures of exposure. Dynamic traffic noise prediction models that include instantaneous vehicle noise emissions can be used to estimate either of these measures. However, current state-of-the-art vehicle noise emission models typically consider a single emission law for each vehicle category, whereas measurements show that the variation in noise emission between vehicles within the same category can be considerable. It is essential that the influence of vehicles that are producing significantly more (or less) noise than the average vehicle are taken into account in modelling in order to correctly predict the levels and frequency of occurrence of road traffic noise events, and in particular to calculate indicators that characterize these noise events. Here, an approach for predicting instantaneous sound levels caused by road traffic is presented, which takes into account measured distributions of sound power levels produced by individual vehicles. For the setting of a receiver adjacent to a dual-lane road carrying free flow traffic, the effect of this approach on estimated percentile levels and sound event indicators is investigated.
\end{abstract}

Keywords: Environmental sound, Noise pollution, Road traffic, Microscopic simulation, Noysim, Python PACS: 43.50.Lj, 43.50.Rq

\section{Highlights}

- A road traffic noise prediction model that accounts for vehicle emission distributions is proposed.

- The software is freely available as a Python plugin to the Aimsun microscopic traffic simulator.

- Simulations show that this refined approach affects estimated percentile sound levels by up to $4 \mathrm{~dB}$.

- The influence of the approach on indicators for the number of noise events is illustrated.

\section{Introduction}

Road traffic noise prediction models are usually directed towards the estimation of $L_{\mathrm{Aeq}}$ or derived indicators such as $L_{\mathrm{den}}$. The main reasons are that energy equivalent levels for road traffic streams are relatively simple and robust to estimate, and that they have a reasonable correlation with annoyance [1]. Criterion levels for road traffic noise utilised for mitigation and planning purposes generally use these single-number energy equivalent metrics of the fluctuating road traffic noise signal. As such, they constitute the predominant indicators used in policy and management (see,

\footnotetext{
${ }^{*}$ Corresponding author

Email addresses: bert.decoensel@intec.ugent.be (Bert De Coensel), lex.brown@griffith.edu.au (A. L. Brown), d.tomerini@griffith.edu.au (Deanna Tomerini) 
for example, the Environmental Noise Directive of the European Commission [2]). However, energy equivalent noise descriptors say very little about the pattern of fluctuations of noise levels over time, yet it may be this temporal pattern that is of particular interest with respect to specific dimensions of human health effects that arise from road traffic noise $[3,4]$.

Sleep disturbance is a major health issue associated with transportation noise [5, 6], and the research evidence is that the physiological effect of such noise on human sleep may depend more on the level and number of noise events in traffic streams than on energy equivalent measures [7-9]. Community reaction to sleep disturbance from night-time noise has been highlighted in a review of the increased operations of night-time freight traffic in Australia [10]. That review recognized that community reaction to the noise made by heavy vehicles on urban roadways, particularly late at night when many trucks prefer to travel because of lower levels of congestion, will be a major constraint to the future movement of trucks in urban areas. It also noted that authorities have yet to develop criteria defining acceptable incidence (and magnitude) of traffic-related noise events, and that current noise measurement techniques do not readily accommodate the assessment of the discontinuous nature of the noise of large vehicles travelling at night.

Further, it has been suggested that noise annoyance may also be determined, at least in part, by noise events from road traffic streams [11-13]. When noise events are noticed, they may distract attention and interfere with activities [14-16]. Roberts et al. [17], too, note that various studies demonstrate anomalies to the accepted view that it is the energy equivalent level that is important for annoyance responses, postulating that it is the pattern of road traffic noise- the presence of noise peak events - that may be critical, also suggesting that response to road traffic noise may be better assessed by some combination of the conventional continuous noise measures with pattern variables such as the number of noise events. In this context, several indicators for traffic noise annoyance that take into account the fluctuation strength of the sound pressure level (Traffic Noise Index, Noise Pollution Level, Common Noise Index [18] etc.) have variously been proposed. Finally, models for the prediction of road traffic noise are increasingly used for urban planning and soundscape planning, where a notion of the expected strength and occurrence of noise events is essential, for example for estimating the efficiency of measures for masking road traffic noise [19, 20].

Based on the above, it is evident that it is of interest to be able to estimate the occurrence, both in magnitude and number, of noise events that can be expected from road traffic streams. There are traffic situations where this may be of particular interest. For example, arterial roads often cross urban areas where, due to signalized intersections, traffic may not be distributed uniformly over time. Moreover, the traffic stream may contain heavy vehicles. When people live at close distance to such roadways, high vehicle passby levels may result in them noticing these events and/or their sleep disturbance. Energy equivalent levels can be, in these and other circumstances depending on the volume of traffic flow, relatively insensitive to the number and level of individual noise peaks from high-noise-emission vehicles in the road traffic stream.

For noise assessment and management where noise events are likely to be prevalent within road traffic streams, knowledge of the time history of the sound level caused by road traffic is required. A number of traffic noise models have been developed in the past that are suited for predicting the time history of the sound level arising from a traffic stream. Examples are the models developed at INRETS [21, 22], at Ghent University [23, 24] and at EPFL [25]. These models predict the instantaneous contribution of single vehicles to the sound level at a receiver location over time, and account for dynamic effects of vehicular traffic, such as the excess noise emission due to acceleration of vehicles (although limitations still exist because of the relative scarcity of measurement data on emissions during acceleration, and because of the limited accuracy of the estimated traffic variables, in particular acceleration, within dynamic traffic models). This dynamic approach can be used to estimate more advanced indicators, such as indicators that assess the 
impact of traffic light cycles [26-28] or indicators that assess the temporal structure of the sound [29]. Clearly, this approach is a refinement of conventional traffic noise prediction models in which emission calculations are based only on average speeds and intensities [30-32]. However, the state-of-the-art vehicle noise emission models [for example those used in the FHWA Traffic Noise Model or the European Imagine model, 33] still consider a single, prototypical emission law for each vehicle category. This is sufficient for correctly predicting energy equivalent sound levels, as these emission laws are determined on the basis of measurements on a sufficiently large number of vehicles sampled from the vehicle fleet. Note that the Imagine model takes into account factors such as the percentage of vans or the percentage of vehicles having illegal exhaust adjustments, but only as corrections to the prototypical emission law for each vehicle category.

Roadside noise measurements of real traffic streams show that differences in noise emission levels (due to age, maintenance, modifications, driving style etc.), between different vehicles within a single vehicle category (for example, cars, trucks, etc.) can be considerable, and that emission distributions for different vehicle categories overlap considerably [34-36]. Consequently, maximum levels obtained by models that use a single emission law per vehicle category may misrepresent reality. To accurately predict the occurrence and strength of noise events caused by road traffic, the influence of vehicles that are producing more/less noise than the average vehicle has to be taken into account. In Section 2 of this paper, a methodology to do this is outlined. The approach uses individualized vehicle emission laws for predicting the instantaneous overall sound pressure level caused by road traffic. In Can et al. [37], a similar approach, where each vehicle in the simulation is assigned an emission law randomly selected from a small number of emission laws for each vehicle category, has been shown to affect the estimation of the levels of vehicle peaks. In the present work, the approach is taken one step further. A distribution of the sound power levels of individual vehicles, based on maximum levels sampled from a large measurement database for cars and trucks under real operating conditions [34], is used as a correction to the prototypical emission law for each vehicle category [38]. The effect of this approach on estimated energy-equivalent levels, percentile levels and sound event indicators is illustrated in Section 3.

\section{Methodology}

\subsection{Overview}

The proposed approach for predicting the traffic noise level time history near roadways is similar in structure to other dynamic traffic noise prediction models [22, 23], and consists of coupling a microscopic road traffic simulation model with a model for instantaneous vehicle noise emission and a point-to-point sound propagation model. A general overview of the model is shown in Figure 1.

A microscopic simulation of road traffic, in which the movement of individual vehicles is simulated, forms the basis of the proposed approach. Given a road network, vehicle fleet properties and aggregated traffic demand data, a simulation run of such a model provides the instantaneous position, speed and acceleration of each vehicle at each timestep during a predefined simulation period. Subsequently, instantaneous emission spectra for all sources are calculated using a noise emission model. Instead of using prototypical vehicle emission factors to estimate the average noise emission for a vehicle given its category, speed and acceleration, the proposed approach extends existing models by taking into account a realistic distribution of vehicle noise emission levels (greyed box in Figure 1). The latter is implemented by adding a zero-mean distribution (in terms of sound power) of emission corrections to account for vehicle state and effects of driving style (see below). Finally, a point-to-point sound propagation model is used to calculate the sound level history at one or more receiver locations. In this paper we model a simplified setting of a 


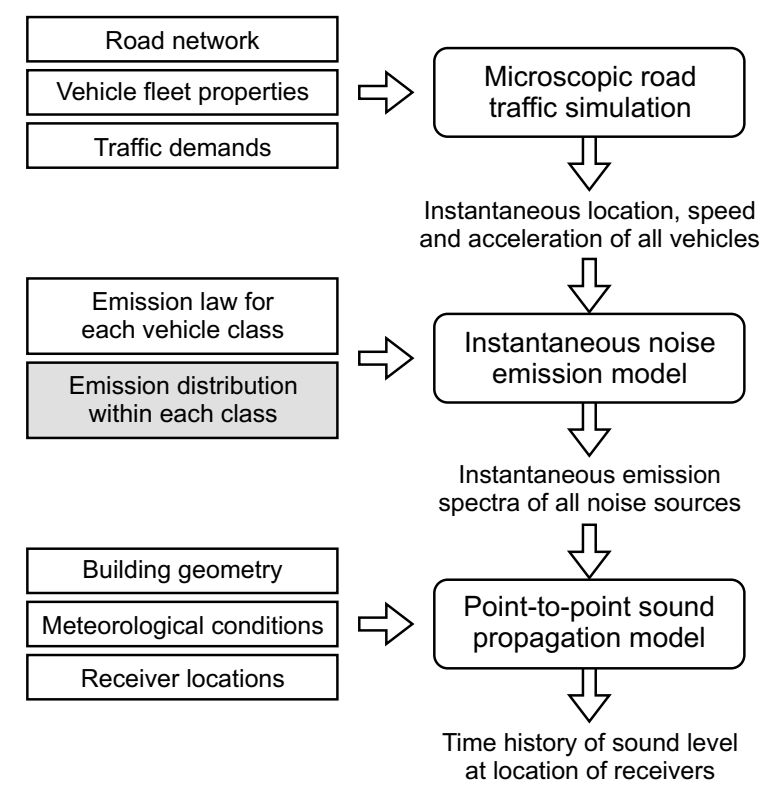

Figure 1: General overview of the proposed methodology. The left column shows the inputs; the right column shows the models applied and the outputs. The greyed box marks the difference between the proposed model and existing models (for present purposes, this paper considers a simplified propagation model with no buildings and fixed meteorological conditions).

receiver adjacent to a dual-lane roadway with unobstructed propagation, but a propagation model could take into account the geometry of the environment, such as the location of buildings or noise barriers, and different meteorological conditions [39].

\subsection{Instantaneous vehicle noise emission model}

To estimate instantaneous point source sound power levels, in this work the Imagine road traffic noise emission model [33] is used, in which two sources of noise are considered separately: rolling noise (generated by tire-road interaction and aerodynamic drag) and propulsion noise (generated by the powertrain and the exhaust). These contributions are given, respectively, by the following formulae:

$$
\begin{gathered}
L_{W, R}(v, a)=A_{R}+B_{R} \cdot \log _{10}\left(\frac{v}{v_{r e f}}\right), \\
L_{W, P}(v, a)=A_{P}+B_{P} \cdot\left(\frac{v-v_{r e f}}{v_{r e f}}\right)+C_{P} \cdot a,
\end{gathered}
$$

where $v$ is the vehicle speed (in $\mathrm{km} / \mathrm{h}$ ) with $v_{\text {ref }}=70 \mathrm{~km} / \mathrm{h}$, and $a$ is the vehicle acceleration (in $\mathrm{m} / \mathrm{s}^{2}$ ). For values of the coefficients $A_{R}, B_{R}, A_{P}, B_{P}$ and $C_{P}$, for each 1/3-octave band and for each vehicle category, we refer to [33]. Both contributions can be aggregated to obtain the total instantaneous sound power level produced by a vehicle, on a 1/3-octave band basis:

$$
L_{W}^{i m}(v, a)=10 \cdot \log _{10}\left(10^{L_{W, R} / 10}+10^{L_{W, P} / 10}\right) .
$$

Note that according to the Imagine road traffic noise emission model [33], for propagation calculations, rolling and propulsion source power is divided over two point sources placed at different heights above the road surface. 
To account for the distribution in sound power emitted by individual vehicles within a given category, as found in actual traffic streams, a correction $\Delta L_{W}$ is added to the point source sound power level calculated using the Imagine model:

$$
L_{W}^{\text {dist }}(v, a)=L_{W}^{i m}(v, a)+\Delta L_{W}
$$

This emission model will be referred to as the "Distribution" model in the remainder of this paper, to distinguish the model from the "Imagine" model that assumes a single emission law for each vehicle category. For each vehicle that enters the simulation, a unique correction $\Delta L_{W}$ is randomly sampled from the measured distributions for the corresponding vehicle category within the Imagine model. For propagation calculations, this correction is applied to both rolling and propulsion noise sources.

\subsection{Construction of correction distributions}

Distributions of the sound power corrections are calculated based on the measurement data reported by Brown \& Tomerini [34]. This study presents distributions of the noise level maxima generated during the pass-by of over 85,000 vehicles in service on urban arterials and motorways in Brisbane (Queensland, Australia). Propagation effects were filtered out, and $L_{\text {Amax }}$ values (measured using "fast" time weighting) were normalized to a distance of $15 \mathrm{~m}$ from the centerline of the vehicle travel path. In order to minimize the effect of the road surface on measurement results, all measurements were conducted near roads with a dense graded asphalt surface, and data from sites where distance effects arising from vehicles travelling on roadways where multiple lanes could have given rise to an error of more than $2 \mathrm{~dB}(\mathrm{~A})$ were removed from the analysis. Consequently, the measured distributions primarily capture the effects of individual driving style, including acceleration volatility [40], and vehicle state. Distributions of $L_{\text {Amax }}$ measured for free-flowing traffic are available for four vehicle categories (cars, non-articulated and articulated trucks, motorcycles), for posted speed limits from 60 to $100 \mathrm{~km} / \mathrm{h}$. Figures 2 and 3 show the distributions of $L_{\text {Amax }}$ used in this work, respectively for cars and articulated trucks (actual counts are plotted to illustrate the sample size for each vehicle category). According to Naish [41], emission spectra between European and Queensland vehicles are similar. Overall, light vehicles in Queensland appear to be slightly louder than their European counterparts, whereas emissions of medium and heavy sized vehicles are again similar. Therefore, it can be assumed that similar distributions would have been obtained if measurements had been carried out elsewhere.

In order to compile distributions of $\Delta L_{W}$ based on the distributions of $L_{\mathrm{Amax}}$, a number of assumptions were considered. Firstly, from a visual inspection of the distributions at different speeds (free-flow conditions), it was concluded that the width and shape of the distribution does not change significantly with speed (i.e. noisy vehicles are equally noisy at different speeds). This allowed pooling the data for different speeds into a single distribution for each vehicle category (as a first-order approximation), taking into account the shift in median value of $L_{\text {Amax }}$ for different speeds. Secondly, it was assumed that corrections are independent of frequency, because no sufficient spectral information was available which could demonstrate otherwise. Thirdly, it was assumed that the distribution in $L_{\mathrm{Amax}}$ equals the distribution in sound power level. Finally, corrections $\Delta L_{W}$ were normalized to a zero mean sound power. This way, energy equivalent sound levels estimated using the proposed Distribution model $L_{W}^{\text {dist }}(v, a)$ would equal those estimated using the Imagine emission model $L_{W}^{i m}(v, a)$. This facilitates comparison between outputs from the two models, in which any systematic differences in noise emission between the European and Australian vehicle fleet are removed [41]. Note that the shape and range of these distributions still characterize the Australian vehicle fleet, but a similar approach can be used to collect locally applicable distributions elsewhere.

Figure 4 shows the final distributions of $\Delta L_{W}$ that are achieved using the approach outlined. These distributions 

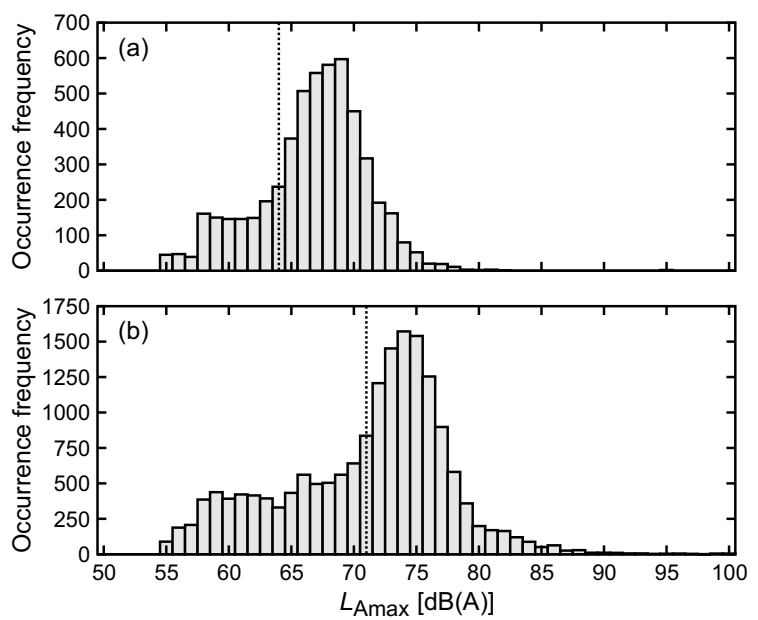

Figure 2: Distributions of the noise level maxima $L_{\mathrm{Amax}}$ of cars, standardized as free-field levels at $15 \mathrm{~m}$ from the centerline of the vehicle travel path. Separate distributions are shown for cars traveling on carriageways with a posted speed limit of (a) $60 \mathrm{~km} / \mathrm{h}, n=5,331$, and (b) $100 \mathrm{~km} / \mathrm{h}, n$ $=17,165$. The dashed line represents the emission of a single car driving at the posted speed limit, with zero acceleration, as calculated using the Imagine road traffic noise emission model (using vehicle category 1 in the Imagine model).
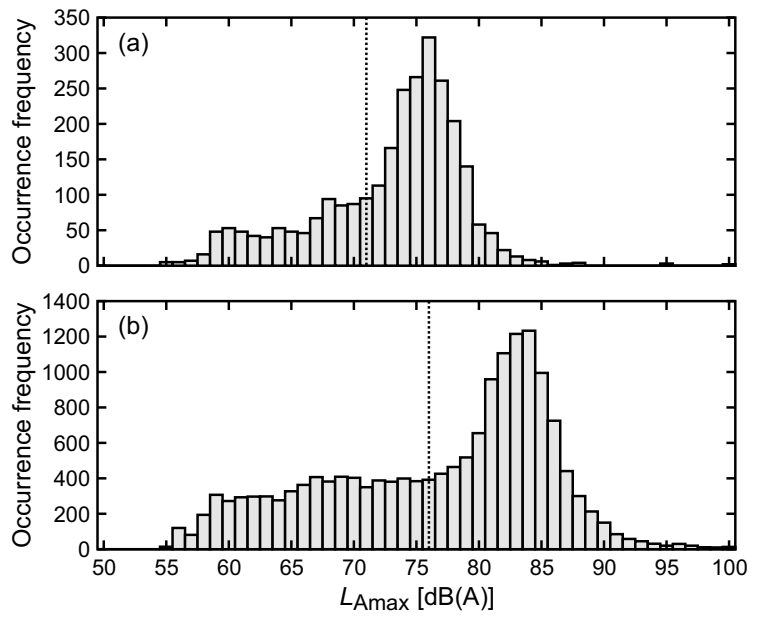

Figure 3: Distributions of the noise level maxima $L_{\mathrm{Amax}}$ of articulated trucks, standardized as free-field levels at $15 \mathrm{~m}$ from the centerline of the vehicle travel path. Separate distributions are shown for articulated trucks traveling on carriageways with a posted speed limit of (a) $60 \mathrm{~km} / \mathrm{h}, n=$ 2,736, and (b) $100 \mathrm{~km} / \mathrm{h}, n=16,475$. The dashed line represents the emission of a single articulated truck driving at the posted speed limit, with zero acceleration, as calculated using the Imagine road traffic noise emission model (using vehicle category 3 in the Imagine model). 

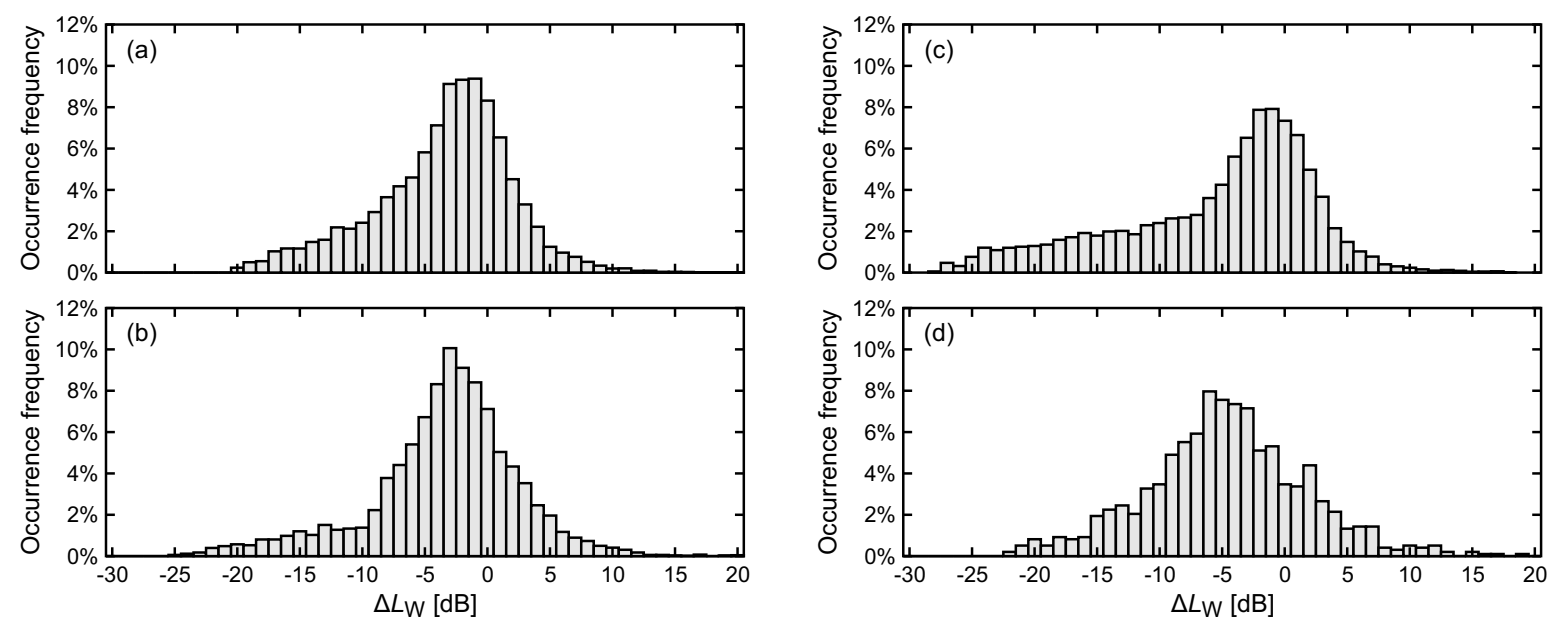

Figure 4: Distributions of the sound power level, relative to the values provided by the Imagine emission model, emitted by (a) cars, (b) nonarticulated trucks, (c) articulated trucks, and (d) motorcycles.

tend to be skewed to lower values for all vehicle categories except for motorcycles. The width of the main peak of the distributions is about 8 to $10 \mathrm{~dB}$, which is very similar to results from measurements performed in the UK [35] and Switzerland [36].

\subsection{Implementation}

The above has been implemented as a simulation framework, called Noysim2, written in the Python programming language (www.python.org). Any microscopic traffic simulation model that provides instantaneous vehicle positions, speeds and accelerations as output can be used with Noysim2, but functionality is provided such that Noysim2 can be readily used as a plugin for Aimsun (www.aimsun.com), a commercially available microscopic traffic simulation software package. Similarly, Noysim 2 can be interfaced with any point-to-point propagation model, but functionality for using the ISO 9613 propagation model [42] is included in the framework and is used in this paper. As an illustration, Figure 5 shows a typical sound level time history, calculated with and without the proposed distribution of corrections. As expected, the time history calculated using the Distribution model shows a large dynamic range in passby levels ( $17 \mathrm{~dB}$ for light vehicles, $25 \mathrm{~dB}$ for heavy vehicles), for the 10 -minute time series considered.

\section{Case study}

\subsection{Road network and simulation scenarios}

The aim of the simulations performed in this case study is to investigate the influence of accounting for realistic vehicle sound power distributions on the estimation of measures that characterize sound events. For this, the simplified setting of a receiver along a straight, one-way, dual-lane road carrying free flow traffic is considered, a setting typically encountered in rural areas. A simulation network containing a single road segment with a length of $2200 \mathrm{~m}$ was constructed using the Aimsun traffic simulation software package (www.aimsun.com). A schematic drawing is shown in Figure 6 (note that a two-way road with one lane in each direction can be expected to lead to the same results). Obviously, it is not feasible to simulate all conceivable road network configurations. However, although the current setting is relatively simple and neglects the potential effects of congestion, the presence of traffic lights etc., it already 

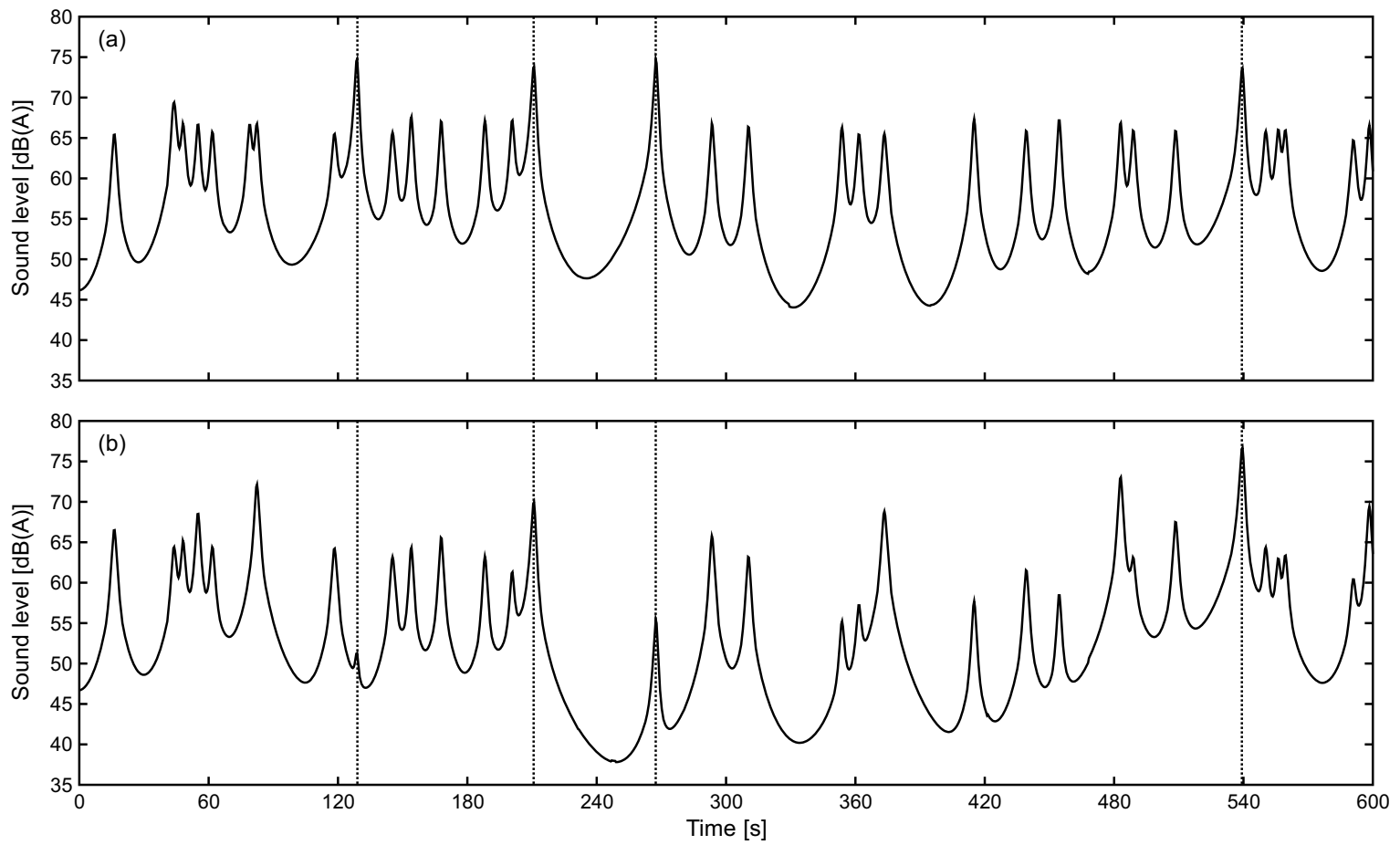

Figure 5: Time history of the sound level at a distance of $15 \mathrm{~m}$ from a two-lane road with a speed limit of $60 \mathrm{~km} / \mathrm{h}$, and a traffic volume of 200 vehicles/h, of which $10 \%$ heavy vehicles, calculated using (a) the Imagine emission model and (b) the Distribution emission model. The same traffic simulation was used for both curves; the dashed lines match the passby of heavy vehicles. 


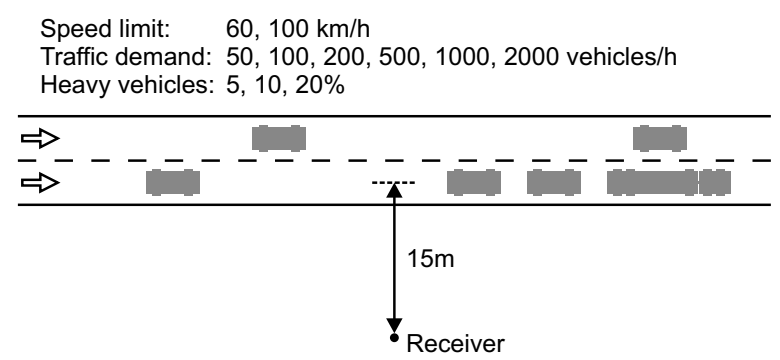

Figure 6: Schematic drawing of the simplified road network of the case study, together with the parameter values for the simulations.

provides some insight in the effects of using an emission distribution on estimated sound event measures. A series of scenarios was created by varying the speed limit, the traffic demand and the percentage of heavy vehicles, as shown in Figure 6. The total number of unique scenarios equals $2 \times 6 \times 3=36$. In order to achieve statistically relevant results, the simulation duration considered was $24 \mathrm{~h}$, with a simulation timestep of $0.4 \mathrm{~s}$. The actual simulations included an additional warm-up period of 5 minutes. Vehicles are loaded onto the network at the entry of the road segment, randomly distributed in time according to a negative exponential distribution.

Instantaneous vehicle noise emission spectra are calculated for all simulation scenarios using the Imagine and the Distribution emission models, based on the same traffic simulation run for both models. For the Imagine emission model, default road surface parameters are used. Subsequently, the time history of the sound level at a receiver located along the perpendicular bisector of the simulated road segment (i.e. near the center of the road segment), at a distance of $15 \mathrm{~m}$ to the centerline of the closest lane, and at a height of $2 \mathrm{~m}$, is calculated. The instantaneous sound level is calculated using the ISO 9613 propagation model [42], assuming free field propagation conditions, default meteorological conditions and a hard surface throughout the simulated area. Finally, energy equivalent levels, percentile levels and sound event indicators are calculated based on the time history of the sound level. Due to the stochastic nature of the sampling used in the Distribution model (in other words, the model randomly selects a correction $\Delta L_{W}$ for each vehicle that is loaded onto the network, according to the appropriate correction distribution for the vehicle category), results may differ between simulation runs. Variability between simulation runs can be expected to decrease with increasing traffic volume. Therefore, a number of simulation runs are performed for the Distribution model, ranging from ten runs for the lowest traffic volume to two runs for the highest traffic volume, and results are averaged. The number of runs for each scenario was chosen such that the total simulation time was more or less the same in all scenarios.

\subsection{Results}

Table 1 shows a first set of acoustical measures calculated for the case study scenarios using the Distribution emission model (averaged arithmetically across simulation runs; the standard deviations are given), and in parentheses, the difference with the Imagine emission model. As expected, there is no significant difference between both models when it comes to predicting $L_{\mathrm{Aeq}, 24 \mathrm{~h}}$ because the corrections in the Distribution model were constructed to give a mean emission sound power equal to that of the Imagine model. The standard deviation in estimates of $L_{\text {Aeq,24h }}$ between simulation runs is found to be smaller than $0.7 \mathrm{~dB}$ in all scenarios.

However, using the Distribution emission model results in estimates for the maximum sound level, $L_{\text {Amax }}$ (calculated over the 24-hour simulation period), that are 12 to $18 \mathrm{~dB}$ higher than those obtained using the Imagine emission 
model. In general, the higher the traffic volume, the larger the difference becomes. Furthermore, the standard deviation in estimates of $L_{\mathrm{Amax}}$ between 24-hour simulation runs can be higher than $3 \mathrm{~dB}$ when the Distribution model is used. This resembles the actual situation, where the maximum sound level is determined by the passage of vehicles at the extreme end of the sound emission distribution, but which are also very rare. The Distribution model is able to take into account the influence of these loud vehicles, but at the cost of the need for much longer simulation times, in order to get stable estimates. Moreover, because of the variability in estimates (and measurements) of $L_{\mathrm{Amax}}$, the use of this indicator for characterizing sound events can be questioned.

When estimates of the percentile levels $L_{\mathrm{A} 01}$ and $L_{\mathrm{A} 05}$ that characterize the average level of sound events are considered, one finds that using the Distribution emission model results in lower values for low traffic volumes, and higher values for high traffic volumes, as compared to using the Imagine emission model. Differences range from $-1.5 \mathrm{~dB}$ to as much as $+3.8 \mathrm{~dB}$ for $L_{\mathrm{A} 01}$, and from $-2.4 \mathrm{~dB}$ to $+0.8 \mathrm{~dB}$ for $L_{\mathrm{A} 05}$. Standard deviations between runs are within $0.4 \mathrm{~dB}$ for all scenarios.

Table 2 shows a second set of acoustical measures calculated for the case study scenarios. The median level $L_{\mathrm{A} 50}$ is consistently estimated to be lower by 1 to $4 \mathrm{~dB}$ when using the Distribution emission model, as compared to using the Imagine model. This is a direct consequence of the correction distributions being skewed to lower values. The effect is largest at low traffic volume.

Finally, three indicators that count the number of sound events in a traffic noise signal are considered. The first indicator, $\mathrm{N}_{\mathrm{CN}}$, measures the number of times that the sound level exceeds $L_{\mathrm{A} 50}$ with at least $3 \mathrm{~dB}$, for at least 3 seconds [43]. This indicator is adaptive, as the threshold for event detection rises with the median level. The second indicator, MM60, uses a fixed value of $60 \mathrm{~dB}(\mathrm{~A})$ as the threshold for event detection, but only takes into account events that are separated in time by at least 3 seconds, and for which the sound level increased with at least $5 \mathrm{~dB}$ within the 25 seconds preceding the event [34]. The third indicator, MM70, is defined in a similar way, but with a threshold for event detection of $70 \mathrm{~dB}(\mathrm{~A})$ instead. In Table 2, values for these indicators are given per hour.

For low traffic volumes, values for $\mathrm{N}_{\mathrm{CN}}$ obtained using the Distribution model do not differ significantly from those obtained using the Imagine model. For intermediate traffic volumes, the Distribution model gives rise to slightly lower values, as sound events produced by the passby of quieter vehicles have a larger chance to be masked by the noise from other louder vehicles in the traffic stream. For high traffic volumes, the interpretation of the results is more complex. Using the Imagine model results in a quasi-constant sound level for high traffic volumes, where only the events caused by a fraction of the heavy vehicles emerge more than $3 \mathrm{~dB}$ above the adaptive threshold, as there are typically several vehicles impacting the sound level at a given time step. The Distribution model gives rise to considerably higher values for $\mathrm{N}_{\mathrm{CN}}$. This comes as no surprise, since the use of the Distribution emission model results in a larger dynamic range for the sound level, and also the noisiest light vehicles give rise to sound events in this case.

For the non-adaptive event indicator MM60, values obtained using the Distribution model are consistently lower than those obtained using the Imagine model, because the quieter vehicles of the Distribution model do not produce sound events that exceed $60 \mathrm{~dB}(\mathrm{~A})$, as can be seen in Figure 5(b). However, such a result is highly sensitive to the particular threshold level chosen. If $70 \mathrm{~dB}(\mathrm{~A})$ is chosen instead, the results are reversed for the low speed limit and for low traffic volumes, with the Distribution model producing more counts than the Imagine model. In these cases, only heavy vehicles produce peaks using the Imagine model. For the high speed limit, values obtained using the Distribution model are again consistently lower than those obtained using the Imagine model, and this is even more pronounced than for MM60, because the correction distributions for heavy vehicles are skewed to lower values. 


\section{Discussion}

\subsection{Effects on noise indicator estimates}

Because the correction distributions have, for the case study described in this work, been normalized to a zero mean sound power, energy equivalent sound levels estimated using the Distribution emission model equal those estimated using the Imagine emission model. However, for the often encountered setting of a receiver adjacent to a dual-lane road, the distribution approach was shown to result in considerably different values for percentile levels as compared to using a single emission law for each vehicle category. In particular, values for the median level $L_{\mathrm{A} 50}$ were consistently estimated to be lower by several $\mathrm{dB}$, a consequence of the the correction distributions being skewed to lower values.

Whereas the distributed emission level approach resulted in stable estimates of percentile levels, estimates of $L_{\mathrm{Amax}}$ were found to be unstable, i.e. not reproducible. When using the Imagine vehicle sound power levels, simulations produce levels of the indicator $L_{\mathrm{Amax}}$ that are stable and predictable across different flow rates, simply because the maximum level emitted by a passing vehicle of a particular type will, unrealistically, be the same irrespective of the vehicle flow rate on the roadway (the level at the receiver may increase slightly with flow rate because of the contributions from other vehicles in proximity to the source vehicle as traffic volume increases). Within the current, more realistic, approach with variable emission levels within each vehicle category, a relatively small sample is drawn from a distribution with a high variance, resulting in instability in the estimates of the $L_{\mathrm{Amax}}$ indicator.

\subsection{Implications for practice and policy}

The finding of instability in the $L_{\mathrm{Amax}}$ indicator has important practical ramifications for measurement practice and for policy. Application of this indicator is allowed, even encouraged, in the Environmental Noise Directive of the European Commission [2] and is reported in most research on sleep disturbance. However, the simulations reported above show that there is no way to undertake a measurement of $L_{\mathrm{Amax}}$ of road traffic streams (particularly for low to moderate traffic volumes) that can produce replicable results. Contiguous $L_{\mathrm{Aeq}}$ measurements for relatively short periods of road traffic are usually consistent (as are replicate measurements on different days of the week), but this is not the case for $L_{\mathrm{Amax}}$. Instability in measurements of $L_{\mathrm{Amax}}$ has been reported previously [44, 45], and is often attributed to traffic related sources such as sirens or claxons [46]. The simulation results in this paper demonstrate that the reason for this instability is, at least partly, due to the variance in vehicle sound power levels. This has ramifications for any policy attempt to set limit values on the $L_{\mathrm{Amax}}$ indicator for the assessment of sleep disturbance. In particular, this will be the case in locations where there are relatively low vehicle flow rates. Many roadways carry lower vehicle flow rates at the times when people are sleeping. Given the above, one can conclude that for characterizing sound events, a more stable indicator than $L_{\mathrm{Amax}}$ is needed.

Another important implication is that measures for reducing traffic noise levels that aim at removing the loudest vehicles from the traffic stream will be much more effective than traffic management measures that target average vehicle speeds, such as lowering speed limits or introducing traffic signal coordination [47].

\subsection{Counting of noise events}

The distributed vehicle emission approach to simulation gives insights into the dynamics of road traffic noise by providing a realistic temporal pattern of noise levels that can be further analyzed using sound event indicators. Three indicators for the number of events, one with and two without an adaptive threshold, were shown to be influenced by traffic flow rates in a different way when using a distribution emission model. The indicator with adaptive threshold $\left(\mathrm{N}_{\mathrm{CN}}\right)$ has its highest values for medium traffic flow rates, whereas the indicators with fixed threshold (MM60, MM70) 
increase monotonically with traffic flow rate, at least for the range considered in this work. Thus, the definition used for sound event delimitation and counting is critical and needs careful consideration.

More importantly, noise scenarios which differ in speed limit, traffic flow rate and percentage of heavy vehicles, may calculate to the same $L_{\text {Aeq }}$ value, but differ substantially in the number of noise events (as counted with any of the three indicators considered) and thus in their effects on people. For example, the scenario with a speed limit of $60 \mathrm{~km} / \mathrm{h}$ and a traffic volume of 2000 vehicles/h (with $5 \%$ heavy vehicles) results in an $L_{\text {Aeq }}$ of $69.2 \mathrm{~dB}(\mathrm{~A})$ at a distance of $15 \mathrm{~m}$ from the road, and in values 84, 295 and 175 for $\mathrm{N}_{\mathrm{CN}}$, MM60 and MM70 respectively. The scenario with a speed limit of $100 \mathrm{~km} / \mathrm{h}$ and a traffic volume of 500 vehicles/h (with $20 \%$ heavy vehicles) results in a similar $L_{\mathrm{Aeq}}$ of $69.1 \mathrm{~dB}(\mathrm{~A})$ at the same distance, but with values of 124, 293 and 220 for $\mathrm{N}_{\mathrm{CN}}$, MM60 and MM70 respectively. Values for MM60 are stable for these different scenarios, but values for $\mathrm{N}_{\mathrm{CN}}$ and MM70 are very different.

\subsection{Limitations}

It has to be noted that the distributions of the sound power corrections that are used in this paper are based on measurement data obtained along urban arterial roads under free flow conditions, as reported in a previous study [34]. Therein, care was taken to filter out the effects of sound propagation, road surface and multiple lanes, such that the source of the variance in the measured sound power level distributions for vehicles within the same category primarily reflected the effects on vehicle emission levels of vehicle state (age, maintenance, modifications) and individual driving style (such as gear-changing behaviour and acceleration volatility [40]). The speeds used for constructing the emission corrections in this work are the posted speed limits, not the actual vehicle speeds. The latter would have varied around the posted speed limits for the measurement locations considered in the previous study [34]. However, because no measurements of actual vehicle speeds or sound spectra were available from the previous study, it was not possible to make a distinction between the effect of the speed distribution and the effect of genuinely louder/quiter vehicles for a given speed (including the effect of driving behavior). Moreover, as only free flow conditions were considered in this work, acceleration values would have been very small or even null. There is a possibility that the distributions of the sound power corrections depend on acceleration; those vehicles that exhibit the highest accelerations might be prone to be the same ones that are the loudest, in which case fighting such behavior would be a very efficient measure to reduce traffic noise levels. Unfortunately, no data was available to confirm or refute this hypothesis.

It can be shown that, for free flow traffic, the speed distribution for a given category of vehicles approximates to a normal distribution, with the width of the distribution related to the mean speed [48]. For example, for a posted speed limit of $100 \mathrm{~km} / \mathrm{h}$, a standard deviation in actual speeds no greater than $20 \mathrm{~km} / \mathrm{h}$ can be expected [49]. From the emission laws within the Imagine road traffic noise emission model [33] it can be derived that in such situation, the effect of the speed distribution on noise levels will be relatively small. A variation in speed between $80 \mathrm{~km} / \mathrm{h}$ and $120 \mathrm{~km} / \mathrm{h}$ would result in a variation of $\pm 3 \mathrm{~dB}$ in sound power level emitted by light vehicles, which is considerably smaller than the width of the distributions used in this work. It has already been shown that the effect of the speed distribution on energy equivalent levels is limited to 0.2 to $0.3 \mathrm{~dB}$ [49]. It can therefore be expected that the effect of the variation in individual vehicle speeds will not influence the general conclusions of this work.

Finally, only a relatively simple free flow traffic scenario was considered in the present work, and sound propagation in an environment without buildings was considered, using the ISO 9613 sound propagation model [42]. Applying the approach outlined in this work to more realistic traffic scenarios in urban context, including scenarios in which congestion may occur, and including a more sophisticated propagation model [50, 51], would yield more accurate results over a wider range of conditions, and would allow to estimate sound event indicators in urban context more accurately. 


\section{Conclusions}

This paper reported on a novel approach for simulating instantaneous sound levels caused by road traffic, extending existing modelling approaches by taking into account a distribution of vehicle sound power levels. The approach allows simulation of a more realistic sound level time history at the location of the receiver. This is of importance for the estimation of indicators that characterize the occurrence, both in magnitude and number, of noise events caused by a traffic stream. The effect of this approach on estimated percentile levels and sound event indicators was investigated through a simulation case study. The introduction of a distribution of vehicle sound power levels was shown to affect estimated percentile sound levels by up to $4 \mathrm{~dB}$, depending on vehicle speeds and traffic flow rates. Moreover, the approach resulted in non-reproducible estimates of $L_{\mathrm{Amax}}$, which strongly limits the use of this indicator for characterizing sound events. Finally, three noise event count indicators, which use different rules to delimit sound events, were shown to be influenced in a different way by traffic flow rates when using the distribution emission model approach.

\section{Acknowledgments}

Bert De Coensel is a postdoctoral fellow of the Research Foundation-Flanders (FWO-Vlaanderen); the support of this organisation is gratefully acknowledged. This work was partly supported by an Australian Research Council Linkage Projects funding scheme (project number LP0990541) with the Department of Main Roads (Queensland) as the industry partner.

\section{References}

[1] H. M. E. Miedema, Relationship between exposure to multiple noise sources and noise annoyance, J. Acoust. Soc. Am. 116 (2) (2004) 949-957.

[2] Directive 2002/49/EC of the European Parliament and of the Council of 25 June 2002 relating to the assessment and management of environmental noise, Environmental Noise Directive, European Commission, Brussels, Belgium (2002).

[3] L. Fritschi, A. L. Brown, R. Kim, D. Schwela, S. Kephalopoulos (Eds.), Burden of disease from environmental noise: quantification of healthy life years lost in Europe, World Health Organization Regional Office for Europe, Copenhagen, Denmark, 2011.

[4] A. L. Brown, Longitudinal annoyance responses to a road traffic noise management strategy that reduced heavy vehicles at night, J. Acoust. Soc. Am. 137 (1) (2015) 165-176.

[5] J. A. Knottnerus, The influence of night-time noise on sleep and health, Publication no. 2004/14E, Health Council of the Netherlands, The Hague, The Netherlands (2004).

[6] A. Carroll, The health effects of environmental noise—other than hearing loss, Publication no. 3311 (JN 7845), enHealth Council, Department of Health and Ageing, Australian Government, Canberra, Australia (2004).

[7] B. Griefahn, A. Marks, S. Robens, Noise emitted from road, rail and air traffic and their effects on sleep, J. Sound Vib. 295 (1-2) (2006) $129-140$.

[8] B. De Coensel, D. Botteldooren, B. Berglund, M. E. Nilsson, T. De Muer, P. Lercher, Experimental investigation of noise annoyance caused by high-speed trains, Acta Acust. Acust. 93 (4) (2007) 589-601.

[9] S. Pirrera, E. De Valck, R. Cluydts, Nocturnal road traffic noise: A review on its assessment and consequences on sleep and health, Environ. Int. 36 (5) (2010) 492-498.

[10] B. Dowdell, Strategies for reducing the environmental impacts of increased night-time operations of road freight: a scoping study, Research report AP-R315/07, Austroads Inc., Sydney, Australia (2007).

[11] M. Björkman, Community noise annoyance: Importance of noise levels and the number of noise events, J. Sound Vib. 151 (3) (1991) 497-503.

[12] T. Sato, T. Yano, M. Björkman, R. Rylander, Road traffic noise annoyance in relation to average noise level, number of events and maximum noise level, J. Sound Vib. 223 (5) (1999) 775-784.

[13] A. L. Brown, An overview of concepts and past findings on noise events and human response to surface transport noise, Proceedings of the 43rd International Congress and Exposition on Noise Control Engineering (Inter-noise), Melbourne, Australia, 2014 
[14] B. De Coensel, D. Botteldooren, T. De Muer, B. Berglund, M. E. Nilsson, P. Lercher, A model for the perception of environmental sound based on notice-events, J. Acoust. Soc. Am. 126 (2) (2009) 656-665.

[15] A. J. Torija, D. P. Ruiz, B. De Coensel, D. Botteldooren, B. Berglund, A. Ramos-Ridao, Relationship between road and railway noise annoyance and overall indoor sound exposure, Transport. Res. D 16 (1) (2011) 15-22.

[16] J. Terroir, B. De Coensel, D. Botteldooren, C. Lavandier, Activity interference caused by traffic noise: experimental determination and modeling of the number of noticed sound events, Acta Acust. Acust. 99 (3) (2013) 389-398.

[17] M. J. Roberts, A. W. Western, M. J. Webber, A theory of patterns of passby noise, J. Sound Vib. 262 (5) (2003) $1047-1056$.

[18] C. Ribeiro, C. Anselme, F. Mietlicki, B. Vincent, R. Da Silva, At the heart of Harmonica project: the Common Noise Index (CNI), Proceedings of the 42nd International Congress and Exposition on Noise Control Engineering (Inter-noise), Innsbruck, Austria, 2013.

[19] B. De Coensel, S. Vanwetswinkel, D. Botteldooren, Effects of natural sounds on the perception of road traffic noise, J. Acoust. Soc. Am. 129 (4) (2011) EL148-EL153.

[20] D. Oldoni, B. De Coensel, M. Boes, M. Rademaker, B. De Baets, T. Van Renterghem, D. Botteldooren, A computational model of auditory attention for use in soundscape research, J. Acoust. Soc. Am. 134 (1) (2013) 852-861.

[21] L. Leclercq, J. Lelong, J. Defrance, Dynamic assessment of road traffic noise: elaboration of a global model, Proceedings of the 18th International Congress on Acoustics (ICA), Kyoto, Japan, 2004, pp. 2785-2788.

[22] A. Can, E. Chevallier, M. Nadji, L. Leclercq, Dynamic traffic modeling for noise impact assessment of traffic strategies, Acta Acust. Acust. 96 (3) (2010) 482-493.

[23] B. De Coensel, T. De Muer, I. Yperman, D. Botteldooren, The influence of traffic flow dynamics on urban soundscapes, Appl. Acoust. 66 (2) (2005) 175-194.

[24] B. De Coensel, D. Botteldooren, F. Vanhove, S. Logghe, Microsimulation based corrections on the road traffic noise emission near intersections, Acta Acust. Acust. 93 (2) (2007) 241-252.

[25] A. Bhaskar, E. Chung, M. Kuwahara, Development and implementation of the areawide Dynamic ROad traffic NoisE (DRONE) simulator, Transport. Res. D 12 (5) (2007) 371-378.

[26] A. Can, L. Leclercq, J. Lelong, J. Lefrance, Accounting for traffic dynamics improves noise assessment: experimental evidence, Appl. Acoust. 70 (6) (2009) 821-829.

[27] A. Can, L. Leclercq, D. Botteldooren, Traffic noise spectrum analysis: dynamic modeling vs. experimental observations, Appl. Acoust. 71 (8) (2010) 764-770.

[28] L. Estévez-Mauriz, J. Forssén, W. Kropp, G. Zachos, Traffic dynamics, road design and noise emission: a case study, Proceedings of the 10th European Congress and Exposition on Noise Control Engineering (Euronoise), Maastricht, The Netherlands, 2015, pp. 1541-1546.

[29] D. Botteldooren, B. De Coensel, T. De Muer, The temporal structure of urban soundscapes, J. Sound Vib. 292 (1) (2006) $105-123$.

[30] M. Madireddy, B. De Coensel, A. Can, B. Degraeuwe, B. Beusen, I. De Vlieger, D. Botteldooren, Assessment of the impact of speed limit reduction and traffic signal coordination on vehicle emissions using an integrated approach, Transport. Res. D 16 (7) (2011) 504-508.

[31] J. Gulliver, D. Morley, D. Vienneau, F. Fabbri, M. Bell, P. Goodman, S. Beevers, D. Dajnak, F. J. Kelly, D. Fecht, Development of an open-source road traffic noise model for exposure assessment, Environ. Modell. Softw. 74 (2015), 183-193.

[32] W. Wei, T. Van Renterghem, B. De Coensel, D. Botteldooren, Dynamic noise mapping: a map-based interpolation between noise measurements with high temporal resolution, Appl. Acoust. 101 (2016) 127-140.

[33] B. Peeters, G. van Blokland, The noise emission model for European road traffic, Technical Report — Deliverable 11 of the Imagine project IMA55TR-060821-MP10, M+P Consulting Engineers, Vught, The Netherlands (2007).

[34] A. L. Brown, D. Tomerini, Distribution of the noise level maxima from the pass-by of vehicles in urban road traffic streams, Road Transp. Res. 20 (3) (2011) 50-63.

[35] G. Watts, A preliminary study of the characteristics of noisy vehicles under cruising conditions - results of roadside measurements, Acta Acust. Acust. 98 (2) (2012) 279-285.

[36] K. Heutschi, L. Poulikakos, Noise monitoring of trucks, Proceedings of the 9th European Conference on Noise Control (Euronoise), Prague, Czech Republic, 2012, pp. 487-492.

[37] A. Can, L. Leclercq, J. Lelong, Dynamic urban traffic noise: do individualized emission laws improve estimation?, Proceedings of the 19th International Congress on Acoustics (ICA), Madrid, Spain, 2007.

[38] B. De Coensel, A. L. Brown, D. Tomerini, D. Botteldooren, Modeling road traffic noise using distributions for vehicle sound power level, Proceedings of the 41st International Congress and Exposition on Noise Control Engineering (Inter-noise), New York City, USA, 2012.

[39] S. E. Reed, J. L. Boggs, J. P. Mann, A GIS tool for modeling anthropogenic noise propagation in natural ecosystems, Environ. Modell. Softw. 37 (2012) 1-5.

[40] E. Ericsson, Independent driving pattern factors and their influence on fuel-use and exhaust emission factors, Transport. Res. D 6 (5) (2001) $325-345$ 
[41] D. Naish, A study on the sound power of Queensland road vehicles, Proceedings of the 20th International Congress on Acoustics (ICA), Sydney, Australia, 2010.

[42] Acoustics - Attenuation of sound during propagation outdoors - Part 2: General method of calculation, International Standard ISO 9613-2, International Organization for Standardization, Geneva, Switzerland (1996).

[43] B. De Coensel, D. Botteldooren, The quiet rural soundscape and how to characterize it, Acta Acust. Acust. 92 (6) (2006) $887-897$.

[44] E. F. Ray, Jr., Measurement uncertainty in conducting environmental sound level measurements, Noise Control Eng. J. 48 (1) (2000) 8-15.

[45] J. Alberola, I. H. Flindell, A. J. Bullmore, Variability in road traffic noise levels, Appl. Acoust. 66 (10) (2005) $1180-1195$.

[46] A. J. Torija, D. P. Ruiz, A. Ramos, Obtaining of a factor to describe the anomalous sound events in traffic noise measurements, Proceedings of the 19th International Congress on Acoustics (ICA), Madrid, Spain, 2007.

[47] B. De Coensel, A. Can, B. Degraeuwe, I. De Vlieger, D. Botteldooren, Effects of traffic signal coordination on noise and air pollutant emissions, Environ. Modell. Softw. 35 (1) (2012) 74-83.

[48] D. Helbing, Traffic and related self-driven many-particle systems, Rev. Mod. Phys. 73 (4) (2001) 1067-1141.

[49] G. Watts, D. van Maercke, H. van Leeuwen, R. Barelds, M. Beuving, J. Defrance, H. Jonasson, R. Nota, G. Taraldsen, J. Witte, Effects of speed distributions on the Harmonoise model predictions, Proceedings of the 33rd International Congress and Exposition on Noise Control Engineering (Inter-noise), Prague, Czech Republic, 2004.

[50] P. Pamanikabud, M. Tansatcha, Geographical information system for traffic noise analysis and forecasting with the appearance of barriers, Environ. Modell. Softw. 18 (10) (2003) 959-973.

[51] U. W. Tang, Z. S. Wang, Influences of urban forms on traffic-induced noise and air pollution: Results from a modelling system, Environ. Modell. Softw. 22 (12) (2007) 1750-1764. 
Table 1: Acoustical measures $\left(L_{\mathrm{Aeq}, 24 \mathrm{~h}}, L_{\mathrm{Amax}}, L_{\mathrm{A} 01}, L_{\mathrm{A} 05}\right)$ of the sound level time history, at a distance of $15 \mathrm{~m}$ from the road, for all scenarios using the Distribution emission model. Results are averaged arithmetically across simulation runs; the standard deviation is included. The difference with the Imagine model is shown in parentheses.

\begin{tabular}{|c|c|c|c|c|c|c|}
\hline $\begin{array}{l}\text { Speed } \\
\text { limit }\end{array}$ & $\begin{array}{c}\text { Heavy } \\
\text { vehicles }\end{array}$ & $\begin{array}{c}\text { Demand } \\
\text { [vph] }\end{array}$ & $\begin{array}{l}L_{\mathrm{Aeq}, 24 \mathrm{~h}} \\
{[\mathrm{~dB}(\mathrm{~A})]}\end{array}$ & $\begin{array}{c}L_{\mathrm{Amax}} \\
{[\mathrm{dB}(\mathrm{A})]}\end{array}$ & $\begin{array}{c}L_{\mathrm{A} 01} \\
{[\mathrm{~dB}(\mathrm{~A})]}\end{array}$ & $\begin{array}{c}L_{\mathrm{A} 05} \\
{[\mathrm{~dB}(\mathrm{~A})]}\end{array}$ \\
\hline \multirow[t]{18}{*}{$60 \mathrm{~km} / \mathrm{h}$} & \multirow[t]{6}{*}{$5 \%$} & 50 & $53.7 \pm 0.4(+0.0)$ & $88.4 \pm 2.2(+13.6)$ & $65.7 \pm 0.2(-0.4)$ & $58.4 \pm 0.2(-2.4)$ \\
\hline & & 100 & $56.4 \pm 0.4(-0.0)$ & $89.2 \pm 1.9(+14.2)$ & $67.8 \pm 0.2(+0.9)$ & $61.9 \pm 0.1(-2.2)$ \\
\hline & & 200 & $59.5 \pm 0.3(-0.1)$ & $90.9 \pm 1.8(+14.0)$ & $70.4 \pm 0.2(+1.4)$ & $65.0 \pm 0.1(-1.1)$ \\
\hline & & 500 & $63.4 \pm 0.2(+0.0)$ & $92.5 \pm 2.3(+16.6)$ & $73.3 \pm 0.1(+1.2)$ & $68.4 \pm 0.1(+0.2)$ \\
\hline & & 1000 & $66.4 \pm 0.2(+0.0)$ & $92.7 \pm 2.4(+15.6)$ & $75.6 \pm 0.2(+1.8)$ & $71.0 \pm 0.1(+0.6)$ \\
\hline & & 2000 & $69.2 \pm 0.0(+0.0)$ & $91.9 \pm 0.3(+14.9)$ & $77.5 \pm 0.0(+2.8)$ & $73.4 \pm 0.0(+0.6)$ \\
\hline & \multirow[t]{6}{*}{$10 \%$} & 50 & $54.3 \pm 0.6(+0.0)$ & $88.4 \pm 2.2(+13.6)$ & $66.2 \pm 0.3(-0.1)$ & $58.6 \pm 0.2(-2.3)$ \\
\hline & & 100 & $57.2 \pm 0.5(-0.0)$ & $89.6 \pm 3.4(+14.2)$ & $68.8 \pm 0.2(+0.8)$ & $62.4 \pm 0.1(-2.1)$ \\
\hline & & 200 & $60.2 \pm 0.2(-0.1)$ & $90.2 \pm 1.2(+13.4)$ & $71.5 \pm 0.2(+0.0)$ & $65.6 \pm 0.1(-0.9)$ \\
\hline & & 500 & $64.2 \pm 0.2(+0.0)$ & $91.2 \pm 0.7(+14.2)$ & $74.5 \pm 0.1(+0.9)$ & $69.3 \pm 0.1(+0.1)$ \\
\hline & & 1000 & $67.1 \pm 0.2(+0.1)$ & $92.7 \pm 2.4(+15.6)$ & $76.5 \pm 0.3(+2.1)$ & $72.0 \pm 0.1(-0.0)$ \\
\hline & & 2000 & $69.9 \pm 0.1(+0.0)$ & $93.4 \pm 2.6(+15.9)$ & $78.4 \pm 0.4(+3.2)$ & $74.4 \pm 0.1(+0.3)$ \\
\hline & \multirow[t]{6}{*}{$20 \%$} & 50 & $55.4 \pm 0.5(+0.1)$ & $89.2 \pm 1.3(+12.6)$ & $67.4 \pm 0.3(-0.0)$ & $59.3 \pm 0.2(-2.2)$ \\
\hline & & 100 & $58.5 \pm 0.4(+0.1)$ & $90.5 \pm 2.6(+14.0)$ & $70.4 \pm 0.2(-0.7)$ & $63.4 \pm 0.2(-1.8)$ \\
\hline & & 200 & $61.3 \pm 0.3(-0.0)$ & $91.1 \pm 0.7(+14.4)$ & $72.9 \pm 0.2(-0.2)$ & $66.6 \pm 0.1(-0.7)$ \\
\hline & & 500 & $65.2 \pm 0.2(-0.0)$ & $91.7 \pm 1.6(+14.7)$ & $75.7 \pm 0.2(+1.5)$ & $70.6 \pm 0.1(-0.7)$ \\
\hline & & 1000 & $68.1 \pm 0.1(+0.0)$ & $92.3 \pm 1.4(+14.8)$ & $77.7 \pm 0.1(+2.8)$ & $73.4 \pm 0.1(-0.3)$ \\
\hline & & 2000 & $71.1 \pm 0.0(+0.1)$ & $93.8 \pm 3.0(+15.9)$ & $79.9 \pm 0.1(+3.8)$ & $75.8 \pm 0.1(+0.7)$ \\
\hline \multirow[t]{18}{*}{$100 \mathrm{~km} / \mathrm{h}$} & \multirow[t]{6}{*}{$5 \%$} & 50 & $58.2 \pm 0.6(-0.0)$ & $92.9 \pm 3.7(+12.7)$ & $70.8 \pm 0.2(-1.5)$ & $61.9 \pm 0.2(-2.2)$ \\
\hline & & 100 & $61.0 \pm 0.4(-0.0)$ & $95.2 \pm 2.7(+15.0)$ & $73.0 \pm 0.1(-0.2)$ & $66.2 \pm 0.1(-2.4)$ \\
\hline & & 200 & $64.1 \pm 0.3(-0.1)$ & $97.1 \pm 1.9(+16.6)$ & $75.4 \pm 0.1(+1.1)$ & $69.9 \pm 0.1(-2.0)$ \\
\hline & & 500 & $68.0 \pm 0.2(+0.0)$ & $98.0 \pm 0.8(+16.6)$ & $78.3 \pm 0.1(+2.1)$ & $73.5 \pm 0.1(-0.4)$ \\
\hline & & 1000 & $71.1 \pm 0.2(+0.0)$ & $98.5 \pm 0.6(+16.4)$ & $80.4 \pm 0.2(+2.4)$ & $75.9 \pm 0.1(+0.3)$ \\
\hline & & 2000 & $73.9 \pm 0.1(+0.1)$ & $98.2 \pm 0.0(+15.8)$ & $82.5 \pm 0.1(+3.2)$ & $78.2 \pm 0.1(+0.8)$ \\
\hline & \multirow[t]{6}{*}{$10 \%$} & 50 & $58.5 \pm 0.6(-0.0)$ & $93.3 \pm 3.3(+13.2)$ & $71.0 \pm 0.2(-1.4)$ & $61.9 \pm 0.2(-2.1)$ \\
\hline & & 100 & $61.5 \pm 0.5(-0.0)$ & $95.2 \pm 2.4(+15.0)$ & $73.5 \pm 0.2(-0.1)$ & $66.5 \pm 0.2(-2.4)$ \\
\hline & & 200 & $64.3 \pm 0.3(-0.1)$ & $96.7 \pm 1.4(+15.3)$ & $75.8 \pm 0.1(+1.1)$ & $70.1 \pm 0.1(-2.0)$ \\
\hline & & 500 & $68.4 \pm 0.2(+0.0)$ & $98.2 \pm 1.5(+16.0)$ & $78.8 \pm 0.1(+1.3)$ & $73.9 \pm 0.1(-0.3)$ \\
\hline & & 1000 & $71.4 \pm 0.2(+0.0)$ & $98.8 \pm 1.4(+16.7)$ & $80.9 \pm 0.2(+2.0)$ & $76.4 \pm 0.1(+0.2)$ \\
\hline & & 2000 & $74.3 \pm 0.1(+0.1)$ & $100.8 \pm 0.5(+18.4)$ & $83.0 \pm 0.2(+3.1)$ & $78.8 \pm 0.1(+0.6)$ \\
\hline & \multirow[t]{6}{*}{$20 \%$} & 50 & $59.2 \pm 0.6(+0.1)$ & $94.4 \pm 1.9(+14.5)$ & $71.5 \pm 0.2(-1.2)$ & $62.4 \pm 0.2(-2.2)$ \\
\hline & & 100 & $62.1 \pm 0.5(-0.0)$ & $95.1 \pm 2.9(+14.5)$ & $74.2 \pm 0.2(+0.1)$ & $66.9 \pm 0.1(-2.4)$ \\
\hline & & 200 & $65.2 \pm 0.3(-0.1)$ & $96.8 \pm 2.0(+15.4)$ & $76.9 \pm 0.2(-0.0)$ & $70.8 \pm 0.1(-1.7)$ \\
\hline & & 500 & $69.1 \pm 0.2(+0.0)$ & $99.1 \pm 2.0(+17.1)$ & $79.8 \pm 0.1(+1.2)$ & $74.7 \pm 0.1(-0.3)$ \\
\hline & & 1000 & $72.1 \pm 0.1(+0.1)$ & $98.4 \pm 0.7(+16.1)$ & $81.8 \pm 0.2(+2.4)$ & $77.3 \pm 0.1(-0.1)$ \\
\hline & & 2000 & $75.0 \pm 0.1(+0.1)$ & $100.0 \pm 0.3(+17.3)$ & $83.9 \pm 0.1(+3.5)$ & $79.7 \pm 0.1(+0.5)$ \\
\hline
\end{tabular}


Table 2: Acoustical measures ( $\left.L_{\mathrm{A} 50}, \mathrm{~N}_{\mathrm{CN}}, \mathrm{MM} 60, \mathrm{MM} 70\right)$ of the sound level time history, at a distance of $15 \mathrm{~m}$ from the road, for all scenarios using the Distribution emission model. Results are averaged arithmetically across simulation runs; the standard deviation is included. The difference with the Imagine model is shown in parentheses.

\begin{tabular}{|c|c|c|c|c|c|c|}
\hline $\begin{array}{l}\text { Speed } \\
\text { limit }\end{array}$ & $\begin{array}{c}\text { Heavy } \\
\text { vehicles }\end{array}$ & $\begin{array}{c}\text { Demand } \\
\text { [vph] }\end{array}$ & $\begin{array}{c}L_{\mathrm{A} 50} \\
{[\mathrm{~dB}(\mathrm{~A})]}\end{array}$ & $\begin{array}{c}\mathrm{N}_{\mathrm{CN}} \\
\text { [events/h] }\end{array}$ & $\begin{array}{c}\text { MM60 } \\
\text { [events/h] }\end{array}$ & $\begin{array}{c}\text { MM70 } \\
{[\text { events/h] }}\end{array}$ \\
\hline \multirow[t]{18}{*}{$60 \mathrm{~km} / \mathrm{h}$} & $5 \%$ & 50 & $37.4 \pm 0.2(-3.0)$ & $28.0 \pm 0.5$ & $36.7 \pm 0.5(-27.2 \%)$ & $4.4 \pm 0.4(+77.6 \%)$ \\
\hline & & 100 & $44.2 \pm 0.1(-2.7)$ & $50.4 \pm 0.9$ & $66.5 \pm 0.8(-26.3 \%)$ & $8.1 \pm 0.4(+73.9 \%)$ \\
\hline & & 200 & $50.7 \pm 0.1(-2.6)$ & $88.8 \pm 1.3(-18.6 \%)$ & $121.4 \pm 1.0(-25.1 \%)$ & $17.4 \pm 0.7(+44.0 \%)$ \\
\hline & & 500 & $57.9 \pm 0.1(-2.3)$ & $123.5 \pm 0.5$ & $233.2 \pm 1.2(-22.4 \%)$ & $42.7 \pm 1.0(+44.4 \%)$ \\
\hline & & 1000 & $62.8 \pm 0.0(-2.3)$ & $107.4 \pm 1.3 \quad(+20.7 \%)$ & $301.0 \pm 1.0(-17.3 \%)$ & $91.9 \pm 1.5 \quad(+4.3 \%)$ \\
\hline & & 2000 & $66.7 \pm 0.0(-1.8)$ & $83.9 \pm 2.6(+103.2 \%)$ & $295.4 \pm 1.2 \quad(-5.4 \%)$ & $175.3 \pm 0.5(-21.2 \%)$ \\
\hline & $10 \%$ & 50 & $37.1 \pm 0.2(-3.2)$ & $26.2 \pm 0.5$ & $34.8 \pm 0.7(-26.1 \%)$ & $5.7 \pm 0.5 \quad(+1.7 \%)$ \\
\hline & & 100 & $44.6 \pm 0.1(-2.8)$ & $49.4 \pm 0.9$ & $66.1 \pm 0.9(-25.4 \%)$ & $10.9 \pm 0.5 \quad(+6.1 \%)$ \\
\hline & & 200 & $51.2 \pm 0.1(-2.5)$ & $88.1 \pm 1.6(-19.0 \%)$ & $122.8 \pm 1.0(-24.0 \%)$ & $22.1 \pm 0.6 \quad(+4.4 \%)$ \\
\hline & & 500 & $58.5 \pm 0.1(-2.3)$ & $117.4 \pm 1.0$ & $231.4 \pm 1.4(-20.8 \%)$ & $54.8 \pm 0.9(+2.2 \%)$ \\
\hline & & 1000 & $63.3 \pm 0.0(-2.2)$ & $106.0 \pm 0.7$ & $293.4 \pm 1.5(-14.9 \%)$ & $109.3 \pm 2.0 \quad(-8.5 \%)$ \\
\hline & & 2000 & $67.3 \pm 0.0(-1.7)$ & $91.2 \pm 0.5 \quad(+84.9 \%)$ & $290.9 \pm 2.7 \quad(-3.8 \%)$ & $198.6 \pm 0.5(-19.7 \%)$ \\
\hline & $20 \%$ & 50 & $37.6 \pm 0.2(-3.3)$ & $25.2 \pm 0.4$ & $34.7 \pm 0.5(-25.5 \%)$ & $8.0 \pm 0.4(-19.8 \%)$ \\
\hline & & 100 & $45.6 \pm 0.1(-2.9)$ & $50.0 \pm 0.7$ & $67.1 \pm 0.9(-24.9 \%)$ & $16.0 \pm 0.6(-21.4 \%)$ \\
\hline & & 200 & $51.9 \pm 0.1(-2.5)$ & $84.5 \pm 0.9 \quad(-21.1 \%)$ & $119.2 \pm 0.9(-23.0 \%)$ & $30.2 \pm 0.8(-20.7 \%)$ \\
\hline & & 500 & $59.3 \pm 0.1(-2.3)$ & $110.4 \pm 1.1 \quad(-10.9 \%)$ & $228.1 \pm 1.6(-19.6 \%)$ & $74.2 \pm 1.2(-17.5 \%)$ \\
\hline & & 1000 & $64.1 \pm 0.0(-2.2)$ & $110.3 \pm 1.2 \quad(-8.2 \%)$ & $283.8 \pm 0.8(-14.9 \%)$ & $141.7 \pm 1.4(-22.1 \%)$ \\
\hline & & 2000 & $68.3 \pm 0.0(-1.6)$ & $98.7 \pm 0.3 \quad(+40.3 \%)$ & $292.2 \pm 2.7(-12.6 \%)$ & $237.3 \pm 0.6(-22.5 \%)$ \\
\hline \multirow[t]{18}{*}{$100 \mathrm{~km} / \mathrm{h}$} & $5 \%$ & 50 & $37.6 \pm 0.2(-3.6)$ & $27.5 \pm 0.3$ & $46.8 \pm 0.5 \quad(-8.4 \%)$ & $26.6 \pm 0.7(-47.9 \%)$ \\
\hline & & 100 & $45.9 \pm 0.2(-3.0)$ & $51.7 \pm 0.4$ & $85.8 \pm 0.4 \quad(-8.3 \%)$ & $50.6 \pm 0.9(-45.9 \%)$ \\
\hline & & 200 & $53.3 \pm 0.1(-2.7)$ & $92.6 \pm 1.1 \quad(-14.5 \%)$ & $156.7 \pm 0.7 \quad(-8.8 \%)$ & $97.9 \pm 0.6(-43.1 \%)$ \\
\hline & & 500 & $61.2 \pm 0.0(-2.3)$ & $132.5 \pm 1.0 \quad(+2.2 \%)$ & $301.0 \pm 1.0(-13.1 \%)$ & $215.5 \pm 0.8(-37.7 \%)$ \\
\hline & & 1000 & $66.5 \pm 0.0(-2.4)$ & $118.2 \pm 1.6 \quad(+14.9 \%)$ & $419.6 \pm 0.8(-18.3 \%)$ & $355.4 \pm 1.1(-30.8 \%)$ \\
\hline & & 2000 & $71.0 \pm 0.0(-2.2)$ & $83.4 \pm 1.5(+229.0 \%)$ & $470.3 \pm 1.3(-19.0 \%)$ & $453.3 \pm 1.4(-21.9 \%)$ \\
\hline & $10 \%$ & 50 & $36.9 \pm 0.2(-3.8)$ & $26.3 \pm 0.3$ & $43.8 \pm 0.4 \quad(-7.8 \%)$ & $25.6 \pm 0.5(-46.2 \%)$ \\
\hline & & 100 & $46.3 \pm 0.2(-2.9)$ & $51.9 \pm 0.6$ & $84.6 \pm 0.5 \quad(-8.1 \%)$ & $51.3 \pm 0.9(-44.3 \%)$ \\
\hline & & 200 & $53.2 \pm 0.1(-2.8)$ & $88.9 \pm 1.2(-15.5 \%)$ & $152.6 \pm 0.8 \quad(-8.8 \%)$ & $96.2 \pm 0.8(-42.5 \%)$ \\
\hline & & 500 & $61.5 \pm 0.1(-2.3)$ & $129.9 \pm 1.8$ & $300.6 \pm 1.0(-12.7 \%)$ & $219.9 \pm 1.2(-36.1 \%)$ \\
\hline & & 1000 & $66.8 \pm 0.0(-2.4)$ & $118.1 \pm 0.9 \quad(+14.4 \%)$ & $409.8 \pm 1.3(-16.4 \%)$ & $350.6 \pm 2.1(-28.5 \%)$ \\
\hline & & 2000 & $71.4 \pm 0.0(-2.2)$ & $85.2 \pm 0.5(+199.8 \%)$ & $463.5 \pm 1.0(-16.4 \%)$ & $450.2 \pm 0.2(-18.8 \%)$ \\
\hline & $20 \%$ & 50 & $36.8 \pm 0.2(-4.1)$ & $25.2 \pm 0.3$ & $43.6 \pm 0.3 \quad(-8.2 \%)$ & $26.5 \pm 0.7(-44.3 \%)$ \\
\hline & & 100 & $46.4 \pm 0.1(-3.1)$ & $48.7 \pm 0.7$ & $82.3 \pm 0.6 \quad(-7.6 \%)$ & $51.4 \pm 0.7(-42.3 \%)$ \\
\hline & & 200 & $54.1 \pm 0.1(-2.8)$ & $87.9 \pm 1.0 \quad(-17.2 \%)$ & $154.5 \pm 0.7 \quad(-9.2 \%)$ & $101.5 \pm 0.7(-40.3 \%)$ \\
\hline & & 500 & $62.0 \pm 0.1(-2.3)$ & $124.0 \pm 1.6$ & $293.1 \pm 1.2(-12.7 \%)$ & $220.1 \pm 1.1(-34.4 \%)$ \\
\hline & & 1000 & $67.4 \pm 0.0(-2.3)$ & $118.3 \pm 1.5 \quad(+12.5 \%)$ & $403.3 \pm 0.9(-16.4 \%)$ & $352.8 \pm 1.4(-26.9 \%)$ \\
\hline & & 2000 & $71.9 \pm 0.0(-2.1)$ & $90.3 \pm 1.1(+145.7 \%)$ & $452.7 \pm 2.5(-15.8 \%)$ & $442.8 \pm 2.4(-17.7 \%)$ \\
\hline
\end{tabular}

